\title{
POLYMER -LIPID HYBRID NANOPARTICLES FOR BRAIN TARGETING THROUGH INTRANASAL DELIVERY
}

\author{
Arya Rajeshwar Kamal Kant,* Juyal Vijay \\ Department of Pharmaceutical Sciences, Bhimtal Campus Kumaun University Nainital, Pin code 263136
}

\begin{abstract}
Brain targeting is a difficult task due to various factors; those factors can restricts the entry of drugs into the brain, in present study polymer-lipid hybrid nanoparticles were prepared for targeting carbamazepine into the brain through the intranasal route. Five formulations were successfully prepared using chitosan, stearic acid and glyceryl mono stearate in different ratio. The particles size were found between $78.88-790 \mathrm{~nm}$, the poly dispersibility index were found in the range of $0.273-0.531$, the zeta potential were found to be $-7.1,-11.6,22.3$ for $\mathrm{HN} 1, \mathrm{HN} 2$, HN3 respectively and for formulation HN4 and HN5 it was found as +12.1 and +22.3 . The entrapment efficiency of all the formulations was found between $62.66-88.31 \%$, the in-vitro releases were found in the range of 40-72\%. The in-vivo studies were performed on Wister rats. Formulation HN5 containing higher conc. of chitosan has shown high drug targeting efficiency. The lipid-polymer hybrid nanoparticles have shown the possibility of targeting the brain through intranasal delivery.
\end{abstract}

Keywords: polymer-lipid hybrid Nanoparticles, carbamazepine, brain targeting, chitosan

Article Info: Received 24 June, 2017; Review Completed 23 Aug, 2017; Accepted 23 Aug, 2017; Available online 24 Aug, 2017

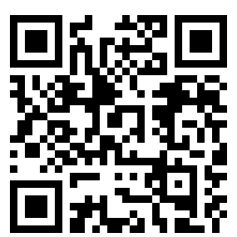

Cite this article as:

Arya RKK, Juyal V, Polymer -lipid hybrid nanoparticles for brain targeting through intranasal delivery, Journal of Drug Delivery and Therapeutics. 2017; 7(4):129-136

DOI: http://dx.doi.org/10.22270/jddt.v7i4.1480

*Address for Correspondence

Rajeshwar Kamal Kant Arya, Department of Pharmaceutical Sciences, Bhimtal Campus Kumaun University Nainital, India- 263136 Email: rajeshwararya@gmail.com

\section{INTRODUCTION}

Carbamazepine is a well-known drug for treating epileptic seizures; it is a first-line drug for the treatment of partial seizures 1, 2, with or without second generalization, and generalized tonic-clonic seizures. The $\mathrm{t}_{1 / 2}$ of carbamazepine is $25-85 \mathrm{hr}$ after a single dose, if dose is repeated for long time, auto-induction takes place in liver, which results in the fluctuations in the plasma conc. this precipitates various unwanted effects like neuromuscular disorder, cardiovascular, gastrointestinal effects and some serious pathological conditions like skin allergies, dysfunction of kidney and liver ${ }^{3,4}$ to overcome these side effects drug should be targeted directly into brain, which is a very tough job because of the anatomy and functionality of a specialized barrier, the blood brain barrier (BBB), the capillary endothelial cells which separate the blood with underlying brain cell, it does not permit the drug or other foreign material to travel into brain from systemic circulation $^{1,5}$. The bioavailability of the particles is found to be the reason for the Nanomedicine to gain precedence. ${ }^{6}$ In last $10-15$ years, various techniques have been employed to deliver drug directly into the brain by penetrating the drug through the BBB. There are various alternative routes for drug administrations are practiced now; intranasal drug delivery has drawn the attention of researcher for development of novel drug delivery ${ }^{5,7}$. Intranasal drug delivery seems good alternative to the injectables for targeting the directly into blood stream or targeting the brain ${ }^{8,9}$. Intranasal route prevents the first pass effect and enzymatic degradation in stomach or intestine, intranasal delivery shows quick onset of action, it is self medicable and can be used in emergency conditions ${ }^{10}$. It has few restrictions like the particle size, molecular weight and low residence time due to rapid 
mucociilary clearance of drugs in the nasal cavity. ${ }^{11,12,13}$ These problems can be reduced by using small carriers like nanoparticles made up of mucoadhesive polymers. These nanoparticles may be used to deliver the drug directly into brain via intranasal administration, because of their nanosize and mucoadhesive characteristics ${ }^{1}$. Polymer-lipid hybrid nanoparticles are new age nanoparticles which are composed of lipid and polymer. Polymer-lipid hybrid nanoparticles are designed to take the advantage of polymeric as well as lipid nanoparticles in a single particle ${ }^{14}$. Jian $\mathbf{L}$ et al., 2010 ${ }^{15}$ developed polymer lipid hybrid nanoparticles containing non-viral gene vector for high transfection efficiency and low toxicity towards normal cells with long circulatory time. Wang J et al., $\mathbf{2 0 1 3}^{16}$ prepared polymer-lipid hybrid nanoparticles for targeting vinculin intracellularlly. In Polymer-lipid hybrid nanoparticles, there two different types of polymer (hydrophilic and lipophilic polymer) are used; therefore a poor water soluble therapeutic agent as well as highly water soluble therapeutic agent can be loaded with high drug loading. The hybrid nanoparticles have good drug loading efficiency. In hybrid nanoparticles the hydrophilic polymeric layer is covered by a lipophilic envelop. Hybrid nanoparticles can also be used for diagnostic purpose ${ }^{17}$, the polymerlipid hybrid nanoparticles have many advantage over the simple polymeric nanoparticles and liposomes. Polymerlipid hybrid nanoparticles can be used in the targeting organs, delivering the genetic materials to the target site 18. The hybrid nanoparticles have the property to adhere with other substrate; it can be easily conjugated with antibody ${ }^{19}$. In our previous work, given elsewhere, we have studied the carbamazepine loaded chitosan nanoparticles, for targeting the brain from intranasal route; the study shows there is a possibility to target the brain from nasal route ${ }^{1}$.

\section{MATERIAL AND METHOD}

\section{Material}

Chitosan was obtained as a gift sample from Central Institute of Fisheries Technology, Kocchi, Carbamazepine was purchased from Sigma Alderich Mumbai, Glyceryl mono stearate, Stearic acid, Tween80 was purchased from $\mathrm{CDH}$, Mumbai, Dialysis membrane-70 was purchased from Hi-Media, Mumbai. All other chemicals and solvents used in the study were of analytical or HPLC grade.

\section{Method}

Polymer-lipid Hybrid nanoparticles containing carbamazepine were prepared by microemulsification followed by ultrasonication method. The lipids (oil phase) were dispersed in n-butanol, and then heated at $10^{\circ} \mathrm{C}$ above the melting point of the lipid. $100 \mathrm{mg}$ drug was dissolved in molten lipid. A $50 \mathrm{ml}$ aqueous solution of chitosan was separately prepared in $0.5 \% \mathrm{v} / \mathrm{v}$ acetic acid and $1 \%$ Tween- 80 and the heated to $70-80^{\circ} \mathrm{C}$. The oil phase was added into hot aqueous phase with continuous stirring for $30 \mathrm{~min}$ to prepare microemulsion. The organic solvent was allowed to evaporate by continuous stirring and heating on mechanical stirrer. The resultant microemulsion was finally poured into $200 \mathrm{ml}$ of ice cold water $\left(2-3^{\circ} \mathrm{C}\right)$. The dispersion was then sonicated by ultrasonic probe sonicator for $9 \mathrm{~min}(3$ cycles of $3 \mathrm{~min}$ ) and off time was $10 \mathrm{sec}$. The resultant dispersion was then centrifuged at 10,000 RPM for 15min. The Polymer-lipid hybrid nanoparticles dispersion was collected and lyophilized.

Table 1: Showing all formulations

\begin{tabular}{|c|c|c|c|c|c|}
\hline \multirow{2}{*}{ Formulation } & \multicolumn{5}{|c|}{ Ingredient } \\
\cline { 2 - 6 } & Carbamazepine & Chitosan & GMS & Stearic acid & Tween-80(ml) \\
\hline H1 & $\mathbf{1 0 0}$ & $\mathbf{1 0 0}$ & $\mathbf{5 0}$ & $\mathbf{5 0}$ & $\mathbf{1}$ \\
\hline H2 & $\mathbf{1 0 0}$ & $\mathbf{1 0 0}$ & $\mathbf{1 0 0}$ & $\mathbf{1 0 0}$ & $\mathbf{1}$ \\
\hline H3 & $\mathbf{1 0 0}$ & $\mathbf{1 0 0}$ & $\mathbf{1 5 0}$ & $\mathbf{1 5 0}$ & $\mathbf{1}$ \\
\hline H4 & $\mathbf{1 0 0}$ & $\mathbf{2 0 0}$ & $\mathbf{5 0}$ & $\mathbf{5 0}$ & $\mathbf{1}$ \\
\hline H5 & $\mathbf{1 0 0}$ & $\mathbf{2 0 0}$ & $\mathbf{5 0}$ & $\mathbf{5 0}$ & 1 \\
\hline
\end{tabular}

\section{Fourier Transform Infrared Spectroscopy ${ }^{20}$}

The FTIR spectra were obtained by FTIR spectrophotometer (FTIR-8400SCE, Shimadzu Corporation). The binary mixture of previously dried powder samples of carbamazepine and excipients were mixed with dry potassium bromide and pellets were made with the help of hydraulic press and scanned within the range of 4000 to $400 \mathrm{~cm}^{-1}$.

\section{Particle Size and Surface Morphology ${ }^{20,21}$}

The particle size and polydispersity index of manufactured carbamazepine containing polymer lipid hybrid nanoparticles was determined with the help of Photon Correlation Spectroscopy using Zetasizer. 3 reading for each sample were recorded. The surface morphology was determined with the help of Transmission Electron Microscopy (TEM).

Zeta Potential: ${ }^{20,21}$

Zeta potential was measured by using Zetasizer (Malvern, Ver. 6.01). The hybrid nanoparticles were diluted 10 times with distilled water and analyzed.

\section{Drug Entrapment Efficiency ${ }^{1}$}

The entrapment efficiency can be calculated by dissolving $25 \mathrm{mg}$ drug loaded nanoparticles in $10 \mathrm{ml}$ methanol and kept for overnight, then filtered by $0.2 \mu$ membrane filter and then analyzed by UV spectrophotometer at $280.4 \mathrm{~nm}$.

$$
\% \mathrm{EE}=\frac{\text { (Initial drug }- \text { Free drug) } 100}{\text { Initial drug }} \quad \text { eq. } 01
$$


Where, Initial drug is the mass of initial drug used for the assay. Free drug is the mass of free drug detected in the supernatant after centrifugation of the aqueous dispersion.

\section{In-Vitro Drug Release Study Nanoparticles ${ }^{1}$}

The in-vitro drug release was carried out on Franz diffusion cell using the dialysis membrane which was mounted over receptor compartment. Phosphate buffer of $\mathrm{pH} 5.5$ was used as the dissolution medium and the temperature of medium was maintained $37^{\circ} \mathrm{C} \pm 0.5^{\circ} \mathrm{C}$. The stirring was done at $100 \mathrm{rpm}$ using a magnetic bar. $5 \mathrm{ml}$ of dissolution medium was withdrawn at $0.5,1,2,3$ to $9 \mathrm{hr}$, which was replaced with the $5 \mathrm{ml}$ fresh medium for maintaining the sink condition. The amount of drug release from the nanoparticles was analyzed by UV spectrophotometer at $280.4 \mathrm{~nm}$.

\section{In-Vitro Drug Release Kinetics ${ }^{22,23}$}

The data obtained from in-vitro drug release were fitted into mathematical models to understand the drug release mechanism, the release data were evaluated with the different kinetics models like zero-order, first-order, Korsmeyer-Peppas and Higuchi using Kinet DS 3 rev 2010 .

\section{In-Vivo study on Rats ${ }^{1},{ }^{2}$}

The in-vivo experiments on rat were performed at Deshpande Lab Bhopal, India. All animal studies were performed according to CPCSEA. The CPCSEA/IAEC approval No. IAEC /DL /2015/RK/012. 54 Male Wister rats were used with a weight range of $200-330 \mathrm{gm}$. The animals were housed in standard cage, in a light controlled room $(14: 10 \mathrm{hr}$. light dark/light cycle) and temperature controlled $20 \pm 2^{\circ} \mathrm{C}$ and $50 \pm 5 \% \mathrm{RH}$, with a proper feeding condition. The food was withdrawn $24 \mathrm{hr}$. before experiment.

\section{Preparation of Drug solution for i.v. Administration $1,2,4$}

The i.v. solution of drug was prepared by dissolving the drug in a mixture of propylene glycol, physiological saline $(0.9 \% \mathrm{NaCl})$-ethanol in a ratio of $5: 3: 2$ to make a final conc. of $1 \mathrm{mg}$ per $\mathrm{ml}$.

\section{Intranasal and i.v. administration ${ }^{1,2,4}$}

The rats anaesthetized by ketamine $(100 \mathrm{mg} / \mathrm{kg}$ ) and xylazine $(10 \mathrm{mg} / \mathrm{kg})$ mixture given by i.p. and the temperature of room were maintained warm. The i.v. solution of drug containing dose of $1 \mathrm{mg} / \mathrm{kg}$ was administered by injection on lateral tail vein. The hybrid nanoparticles were administered at a dose equivalent to $1 \mathrm{mg} / \mathrm{kg}$ to rat. The rat was placed on one side and the formulation was instilled using a polyurethane tube attached to a syringe. The tube inserted to $10 \mathrm{~mm}$ deep into one of the nares, to deliver the formulation to roof of the nasal cavity. The rats were divided into two groups, group 1 containing 18 animals which received the drug by i.v. administration and group 2 containing 36 animals received polymer-lipid hybrid nanoparticles. At a set time interval $(5,15,30,45,60,120 \mathrm{~min})$ after dosing 3 animal per time point were sacrificed by cervical dislocation then decapitated. The blood was then immediately collected in tube containing heparin. The brain was removed and weighed. The blood samples were centrifuged at $4^{0} \mathrm{C}$ at $4000 \mathrm{rpm}$ for $10 \mathrm{~min}$. the plasma were stored at $-30^{\circ} \mathrm{C}$ for further analysis. The brain was homogenized with $0.1 \mathrm{M}$ sod phosphate buffer pH5.0 $(4 \mathrm{ml} / \mathrm{gm})$ of tissue. The Teflon pestle tissue homogenizer was used. Tissue homogenate was then centrifuged at $4000 \mathrm{rpm}$ for $15 \mathrm{~min}$ at $4^{\circ} \mathrm{C}$ and the supernatant was then kept at $-30^{\circ} \mathrm{C}$ for further analysis.

\section{Drug Analysis: ${ }^{1}$}

The analysis of the Carbamazepine was performed by using HPLC with variable wavelength UV detector (SPD- 20A) operated at $280.4 \mathrm{~nm}$. Column used in HPLC is of $250 \mathrm{~mm} \times 4.6 \mathrm{~mm} 5 \mu$ (Enable, C18G, $150 \mathrm{mmx} 4.6 \mathrm{~mm}, 5 \mu$ is suitable) with a flow rate of 1 $\mathrm{ml} / \mathrm{min}$ (isocratic). The mobile phase consists of a blend of methanol /phosphate buffer, (80:20) v/v. The amount of carbamazepine in serum and brain was expressed as $\mathrm{ng} / \mathrm{ml}$ serum.

\section{Pharmacokinetic analysis ${ }^{1}, 2,4$}

The peak plasma conc. $\mathrm{C}_{\max }$ of drug was directly observed from plasma or brain and the time to reach $\mathrm{C}_{\max }\left(\mathrm{T}_{\max }\right)$ was directly estimated from the data received by experiments, other Pharmacokinetic parameter were calculated based on the SEM $(n=3)$ at each time point by a non-compartment pharmacokinetics analysis. The pharmacokinetics parameters were evaluated were the Area Under curve AUC from $t_{0}$ to the last quantifiable conc. $t_{\text {last }}$ by linear trapezoidal rule. For determining the brain targeting efficiency of intranasal drug delivery the drug targeting efficiency (DTE) index was calculated. It is a ratio of nasal and i.v. ${ }^{1}, 2,4$

$$
\mathrm{DTE}=\frac{\left(\mathrm{AUC}_{\text {brain }} / \mathrm{AUC}_{\text {plasma }}\right) \text { intranasal }}{\left(\mathrm{AUC}_{\text {brain }} / \mathrm{AUC}_{\text {plasma }}\right) \text { i.v. }}
$$

Where $\mathrm{AUC}_{\text {brain }}$ and $\mathrm{AUC}_{\text {plasma }}$ are the area under the drug conc. time curve for brain and plasma after intranasal and i.v. application. For the good drug targeting the DTE should be $>1$. The data were expressed as SEM, a comparison was done between i.v. and intranasal delivery, single unpaired one tail ANOVA was used, difference was considered significant for a $\mathrm{p}$ value $\mathrm{p}<0.05$.

\section{RESULT AND DISCUSSION}

\section{Fourier Transform Infrared Spectroscopy}

The FTIR spectra of a-carbamazepine b-physical mixture of carbamazepine, chitosan, glyceryl mono stearate and stearic acid are given in fig.1. The spectra showed characteristic absorption band at 3464 (NH Stretching of NH2) remain unchanged 3464, 3154 (Aromatic $\mathrm{CH}$ stretching) slightly changed to 3159 , $1674(\mathrm{C}=\mathrm{O}$ stretching of $\mathrm{CO} \mathrm{NH} 2)$ slightly changed to 1677 , and $1488(\mathrm{C}=\mathrm{C}$ ring stretching) remain unchanged as 1488. The FTIR spectra of carbamazepine revealed that there was no chemical interaction between carbamazepine and the excipients. 


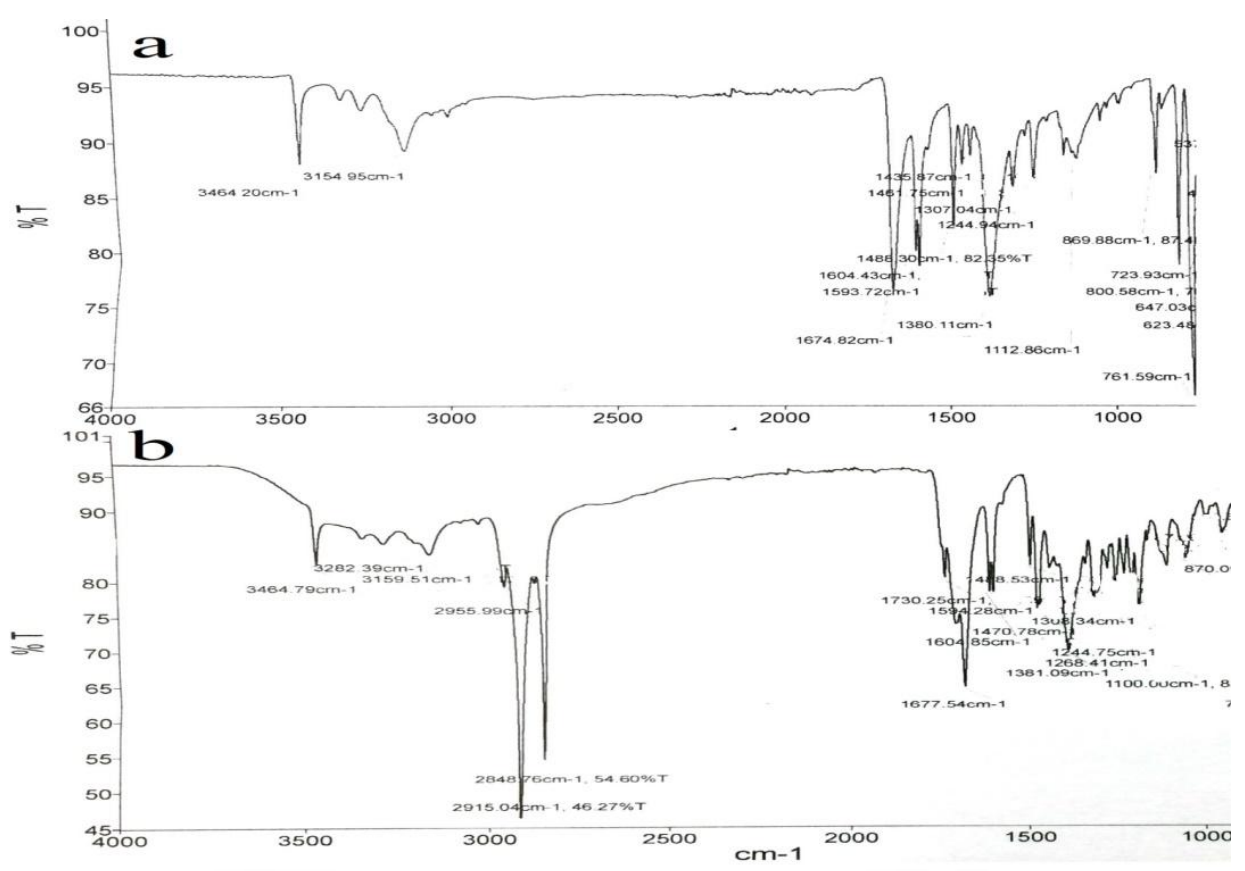

Figure 1: FTIR spectra of a-carbamazepine, b- carbamazepine + stearic acid + chitosan + glycerylmonosterate

\section{Particles Size and Morphology}

The result of particles size and polydispersibility indexes (PDI) of prepared nanoparticles are given in table 2. The particles size ranging from 78.88 to $790 \mathrm{~nm}$. Formulation HN1 contains chitosan and lipid in equal amount, a hazy preparation with a size of $790 \mathrm{~nm}$ was found. In this study, it was observed that, the conc. of lipid influences the size of nanoparticles, on increasing the conc. of lipids, the particle size was decreased and
PDI was increased and then on increasing the conc. of lipid again, the particle size was increased from 87.88 to $125 \mathrm{~nm}$ (formulation $\mathrm{HN} 2$ and $\mathrm{HN} 3$ ). The conc. of chitosan also influences the particles size, as the chitosan conc. was increased the particle size of the nanoparticles also increased.

The TEM study was done to get surface morphology of nanoparticles. The surface of nanoparticles was found rough and somewhat spherical (fig. 2.)

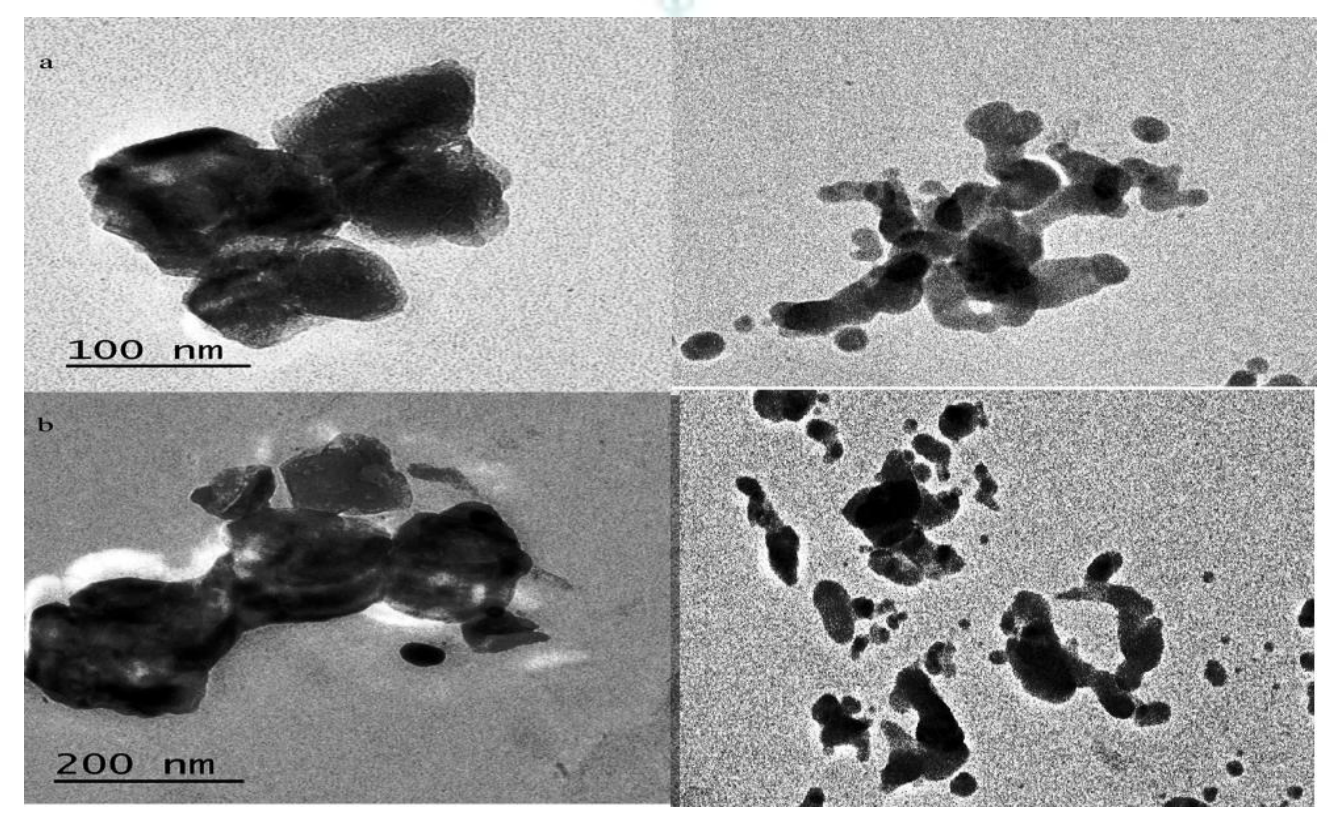

Figure 2: Transmission Electron Microscopic photograph of a-formulation HN2, b-formulation HN4 Zeta potential

\section{Zeta potential}

The zeta potential ranged from -7.1, -11.6, and 22.3 for HN1, HN2, HN3, for HN4 and HN5 zeta potential value were found as +12.1 and +22.3 (table -2 )

\section{Percentage Entrapment Efficiency}

The percentage entrapment efficiency was found to be in the range of $62.66-88.31 \%$ (table- 2). The conc. of polymer and lipid influences the entrapment efficiency, the study reveals that, initially in case of HN1 as the lipid and chitosan conc. were equal the entrapment efficiency was found to be $62.66 \%$ and on increasing the 
conc. of lipid in formulation $\mathrm{HN} 2$, the entrapment efficiency was increased to $85.22 \%$, the then it was decreased to $78.45 \%$ in formulation $\mathrm{HN} 3$, this is due to the formation of a hard and thick layer of lipid, on increasing the conc. of lipid quick quenching of lipid takes place which forms a hard and thick layer, therefore the drug could not penetrate into the lipid layer. But in case of HN4 and HN5 as the conc. of chitosan increased the entrapment efficiency increases again to $86.51 \%$ $88.31 \%$.

Table 2: Results of Particle size, PDI, Zeta potential, Drug content, entrapment efficiency

\begin{tabular}{|c|c|l|c|c|c|}
\hline S.No & Formulation & Particle size (nm) & PDI & Zeta potential (mV) & \% entrapment efficiency \\
\hline $\mathbf{1}$ & HN1 & $780 \pm 1.912$ & $0.531 \pm 0.317$ & -7.1 & 62.66 \\
\hline $\mathbf{2}$ & HN2 & $78.88 \pm 1.21$ & $0.383 \pm 0.256$ & -11.6 & 85.22 \\
\hline $\mathbf{3}$ & HN3 & $135 \pm 2.391$ & $0.632 \pm 0.216$ & -22.3 & 78.45 \\
\hline $\mathbf{4}$ & HN4 & $228 \pm 2.532$ & $0.273 \pm 0.225$ & +12.1 & 86.51 \\
\hline $\mathbf{5}$ & HN5 & $250 \pm 2.390$ & $0.321 \pm 0.214$ & +17.3 & 88.31 \\
\hline
\end{tabular}

\section{In-Vitro Drug Release:}

The in-vitro release was carried out for $9 \mathrm{hr}$ in buffer $\mathrm{pH}$ 5.5 , it was found to be in the range of $40-75 \%$. The observation revealed that formulation $\mathrm{HN} 1, \mathrm{HN} 4$ and HN5 shows the initial burst release of $28-34 \%$ in first $30 \mathrm{~min}$ and then released slowly, the lipid conc. on increasing lipid conc. the drug release was retarded, as in case of formulation $\mathrm{HN} 1, \mathrm{HN} 2$ and $\mathrm{HN} 3$, due to the formation of a thick layer. But when the chitosan conc. increased the drug release also increased in case of formulation HN4 and HN5.

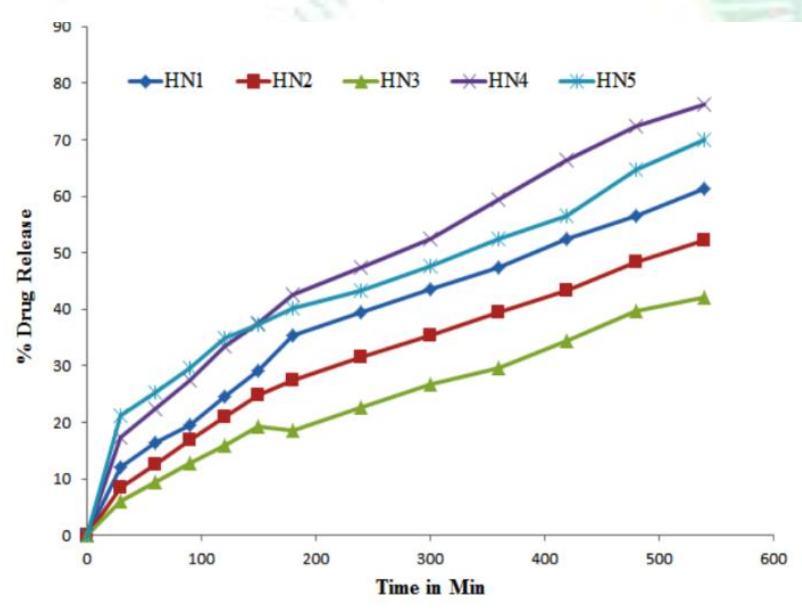

Figure 3: Drug release profile of all Formulation in Phosphate buffer of $\mathrm{pH} 5.5$

The formulation HN5 has shown slow release than HN4 because higher conc. of chitosan retards the release rate after $45 \mathrm{~min}$. because the polymer forms gel after swelling which hinder the release rate. In case of formulation $\mathrm{HN} 1$ the lipid and chitosan conc. is equal, the release was found to be 61.3. In case of HN2 and HN3 where the lipid conc. is more than HN1, the drug release decreased on increasing the conc. of lipid. A significant difference $\mathrm{p}>0.05(\mathrm{p}=0.000256)$ was found. The formulations HN4 and HN5 have higher conc. of chitosan, a significant difference $\mathrm{p}<0.05(0.0335)$ was found in release rate of HN4 and HN5, which shows, as the conc. of chitosan increases the drug release was decreased.

\section{Kinetic modelling}

All the five preparations were evaluated for drug release kinetics using Kinet DS 3 rev 2010 to know the best fit kinetic model. The drug release of formulation HN1 followed the Korsmeyer Peppas release pattern; this is due the presence of both chitosan polymer and lipid in same ratio. The drug release was solely affected by hydrophilic polymer chitosan, this expression tells that drug release follows Korsmeyer model with non-Fickian diffusion super class II $(0.45<\mathrm{n}<0$ 8.9), the fickian case-II transport mechanism associated with formation of water soluble glassy polymer and dissolution of lipid matrix. The drug release from formulation HN2 and HN3 best fit into the Higuchi model, the drug release depended on porosity and tortuosity of lipid matrix, the drug release decreased on increasing the lipid content because the porosity of lipid matrix was decreased.

The drug release from formulation HN4 and HN5 best fit into the Korsmeyer-Peppas model. This shows the drug release was controlled by higher conc. of hydrophilic polymer chitosan, the release pattern of both formulation followed super class II where $0.45<\mathrm{n}<0.89$ case II.

Table 3: Release kinetic parameters of polymer lipid hybrid nanoparticles

\begin{tabular}{|c|c|c|c|c|c|c|c|c|}
\hline Formulation & \multicolumn{2}{|c|}{ Zero order } & \multicolumn{2}{c|}{ First order } & \multicolumn{2}{c|}{ Korsmeyer Peppas } & \multicolumn{2}{c|}{ Higuchi } \\
\hline & $\mathbf{r}^{\mathbf{2}}$ & $\mathbf{K}$ & $\mathbf{r}^{\mathbf{2}}$ & $\mathbf{K}$ & $\mathbf{r}^{\mathbf{2}}$ & $\mathbf{K}$ & $\mathbf{r}^{\mathbf{2}}$ & $\mathbf{K}$ \\
\hline HN1 & 0.9969 & 0.5734 & 0.9968 & -0.0005 & $\mathbf{0 . 9 9 6 9}$ & $\mathbf{0 . 5 7 3 4}$ & 0.9933 & 0.027 \\
\hline HN2 & 0.9272 & 0.0009 & 0.9733 & -0.0009 & 0.992 & 0.5814 & $\mathbf{0 . 9 9 4}$ & $\mathbf{0 . 0 2 1 9}$ \\
\hline HN3 & 0.8904 & 0.0008 & 0.9733 & -0.0009 & 0.986 & 0.556 & $\mathbf{0 . 9 9 4 8}$ & $\mathbf{0 . 0 2 0 4}$ \\
\hline HN4 & 0.9163 & 0.0011 & 0.9846 & -0.0006 & $\mathbf{0 . 9 8 9 9}$ & $\mathbf{0 . 5 0 6 7}$ & 0.9921 & 0.0311 \\
\hline HN5 & 0.8498 & 0.001 & 0.9733 & -0.0009 & $\mathbf{0 . 9 8 7 9}$ & $\mathbf{0 . 3 8 7 8}$ & 0.9868 & 0.0267 \\
\hline
\end{tabular}


Formulation HN2 contains lipid and chitosan in a ratio of 2:1 and HN4 contains lipid and chitosan in a ratio of 1:2 both formulations were selected to understand the behavior and effect of polymers on the biodistribution of drug into brain or plasma from intranasal route. All selected formulation $\mathrm{HN} 2$ and $\mathrm{HN} 4$ was further evaluated for pharmacokinetics studies.

\section{Pharmacokinetics analysis of i.v. intranasally applied hybrid nanoparticles HN2 and HN4}

Fig:4 shows the drug conc. in plasma and brain after i.v. administration, the peak plasma conc. $\left(\mathrm{C}_{\max }\right)$ was found to be 1199 ng which was achieved in plasma in first $5 \mathrm{~min}$ and in brain the $\mathrm{C}_{\max }$ was achieved in 30 min. The $\mathrm{C}_{\max }$ in brain was found to be $1357 \mathrm{ng}$. Initially a higher conc. of drug was achieved in plasma then gradually a decline was seen in plasma conc. whereas the drug conc. in brain was increased slowly, this increment of drug conc. in brain after some time because the drug goes into brain from systemic circulation. The ratio of $\mathrm{AUC}$ (Brain) and AUC (Plasma) was found as 0.7144, this depicts, carbamazepine distributed into brain (target) and plasma (non-target) slight equally. This study also revealed that the drug initially achieve high conc. in plasma, this can lead to side effects.

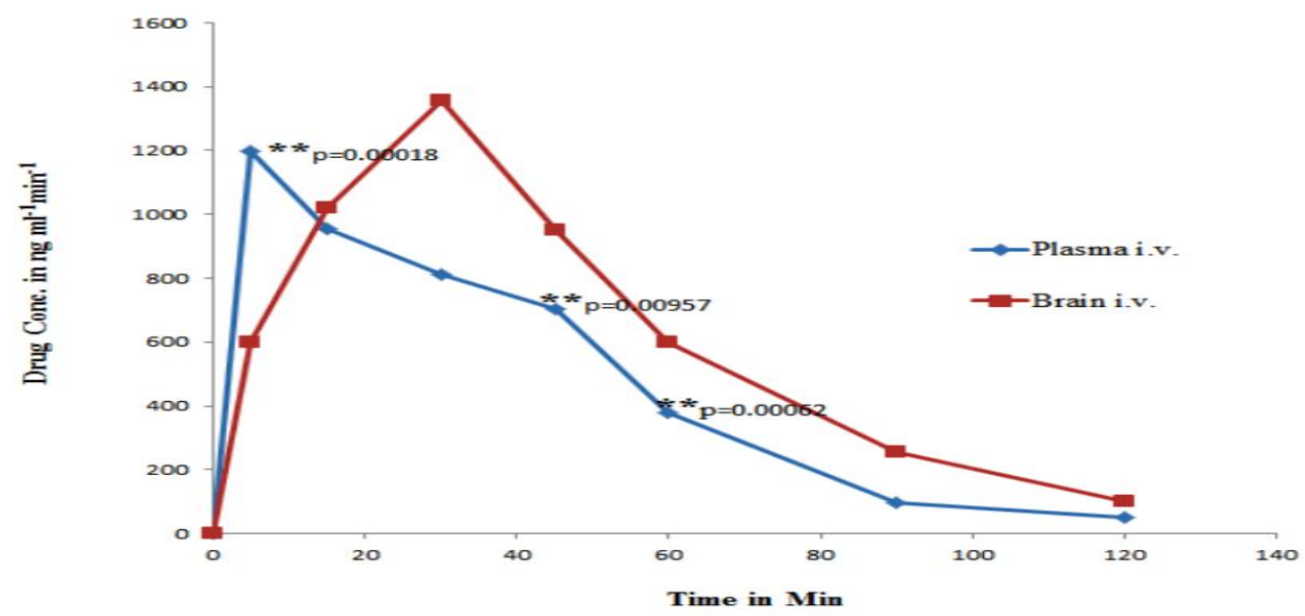

Figure 4: Showing plasma and brain concentration- time profile of carbamazepine after intravenous administration $n=3$ SEM unpaired ANOVA, ** represents a significant difference, $\mathrm{p}<0.05$

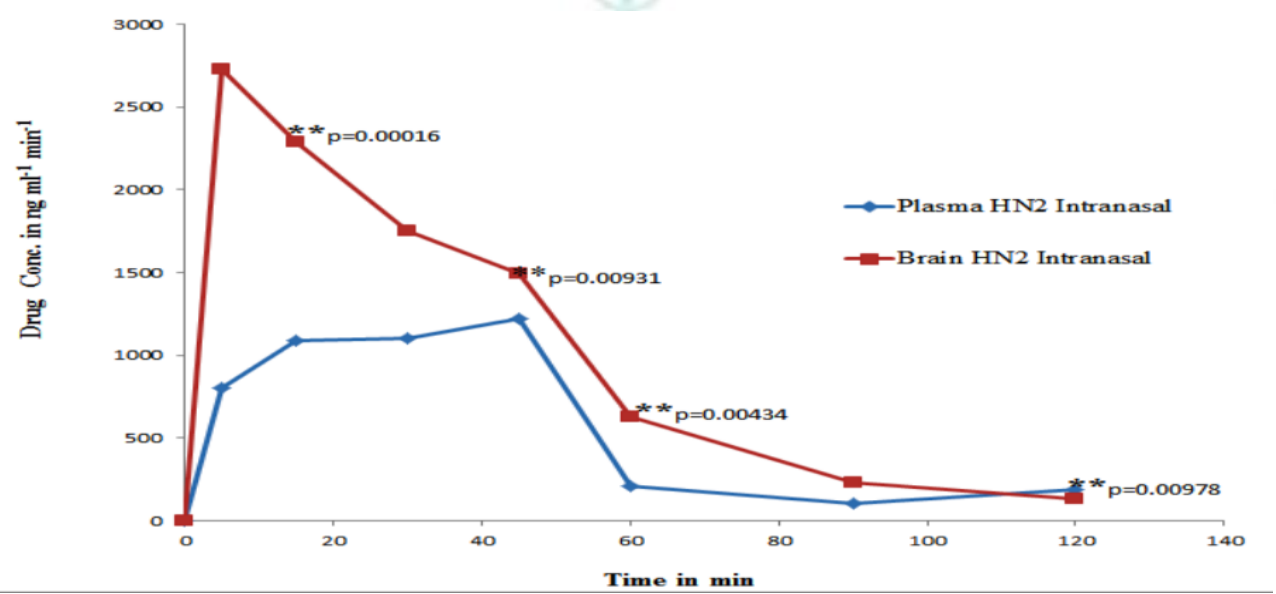

Figure 5: Showing the plasma brain profile of formulation HN2 the conc. of drug ng/ml in the plasma and brain after intranasal administration of formulation $n=3$ SEM unpaired ANOVA, ** represents a significant difference, $p<0.05$.

Figure 5 shows the drug conc. in plasma and brain after intranasal administration of hybrid nanoparticles HN2, The $\mathrm{C}_{\max }$ was found to be 2730ng in brain, which was achieved in first $5 \mathrm{~min}$ but in plasma the $\mathrm{C}_{\max }$ was achieved in $45 \mathrm{~min}$ and the $\mathrm{C}_{\max }$ was found to be $1223 \mathrm{ng}$. The drug conc. in brain declined slowly. The drug conc. in plasma increases slowly and achieved $\mathrm{C}_{\max }$ then the conc. falls suddenly. This shows almost a 2-time increase in $\mathrm{C}_{\max }$ was found in brain than plasma. In all time points a significant difference was found in drug conc. in plasma and brain, The $\mathrm{AUC}_{(\mathrm{Brain})}$ and $\mathrm{AUC}_{\text {(Plasma) }}$ ratio was found as 1.881 , this represents, that the high distribution of drug into brain than plasma can be achieved with HN2 nanoparticles. It shows hybrid nanoparticles target the brain more, rather than plasma. In formulation HN2 the chitosan polymer increases the residence time of the formulation when administered nasally. The brain targeting efficiency DTE was found to be 2.566. This shows hybrid nanoparticles have a 
higher brain targeting efficiency than i.v. administration. The results are summarized in table. 2

Fig: 6 show the drug conc. in plasma and brain after intranasal administration of hybrid nanoparticles HN4. The $\mathrm{C}_{\max }$ was achieved in $5 \mathrm{~min}$ in brain and the $\mathrm{C}_{\max }$ was found to be 3230, but in plasma it has taken 30min to achieve $\mathrm{C}_{\max }$. The $\mathrm{C}_{\max }$ in plasma was found to be 1298ng. The $\mathrm{AUC}_{(\text {Brain) }}$ and $\mathrm{AUC}_{\text {(Plasma) }}$ ratio as 2.996, which shows, the high distribution into brain than plasma can be achieved with hybrid nanoparticles having higher conc. of chitosan. It shows hybrid nanoparticles target the brain rather than plasma, when chitosan was used in twice amount of lipid. Because the chitosan has the mucoadhesive property which enhanced the residence time of formulation in the nasal cavity. The DTE was found to be 3.698. The study shows that formulation $\mathrm{HN} 4$ achieves the highest conc. in brain followed by HN2 then by i.v.

The pharmacokinetic parameter after i.v. and intranasal administration of carbamazepine in plasma and brain are given in table 4 and brain to plasma ratio at different time point is shown in fig. 7.

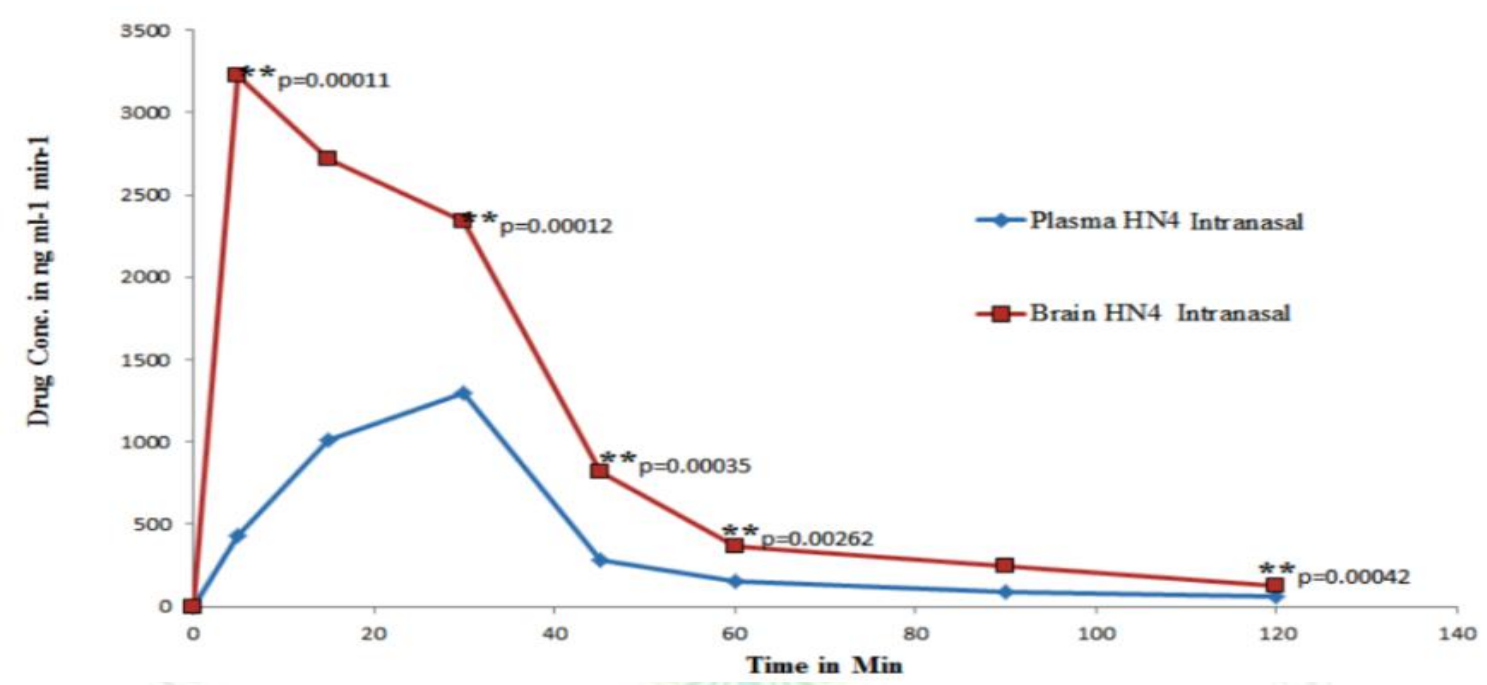

Figure 6: Showing the plasma brain profile of formulation HN4 the conc. Of drug ng/ml in the plasma and brain after intranasal administration of formulation $n=3$ SEM unpaired ANOVA, ** represents a significant difference, $p<0.05$.

Table 4: Showing pharmacokinetic parameter after i.v. and intranasal administration of carbamazepine

\begin{tabular}{|l|l|l|l|l|l|}
\hline Formulation & Organ/tissue & $\mathbf{C}_{\max }$ & $\mathbf{T}_{\max }$ & $\mathbf{A U C}_{\mathbf{1 2 0 m i n}}$ & DTE \\
\hline \multirow{3}{*}{ i.v. } & Blood & $1199 \mathrm{ng}$ & $5 \mathrm{~min}$ & $110.51 \mu \mathrm{gml}^{-1} \mathrm{~min}^{-1}$ & ----- \\
\cline { 2 - 6 } & Brain & $1357 \mathrm{ng}$ & $30 \mathrm{~min}$ & $78.96 \mu \mathrm{gml}^{-1} \mathrm{~min}^{-1}$ & 2.566 \\
\hline \multirow{3}{*}{ HN2 Nasal } & Blood & $1230 \mathrm{ng}$ & $45 \mathrm{~min}$ & $65.850 \mu \mathrm{gml}^{-1} \mathrm{~min}^{-1}$ & \\
\cline { 2 - 5 } & Brain & $2730 \mathrm{ng}$ & $5 \mathrm{~min}$ & $120.740 \mu \mathrm{gml}^{-1} \mathrm{~min}^{-1}$ & 3.69 \\
\cline { 2 - 5 } & Blood & $1298 \mathrm{ng}$ & $5 \mathrm{~min}$ & $46.592 \mu \mathrm{gml}^{-1} \mathrm{~min}^{-1}$ & \\
\hline
\end{tabular}

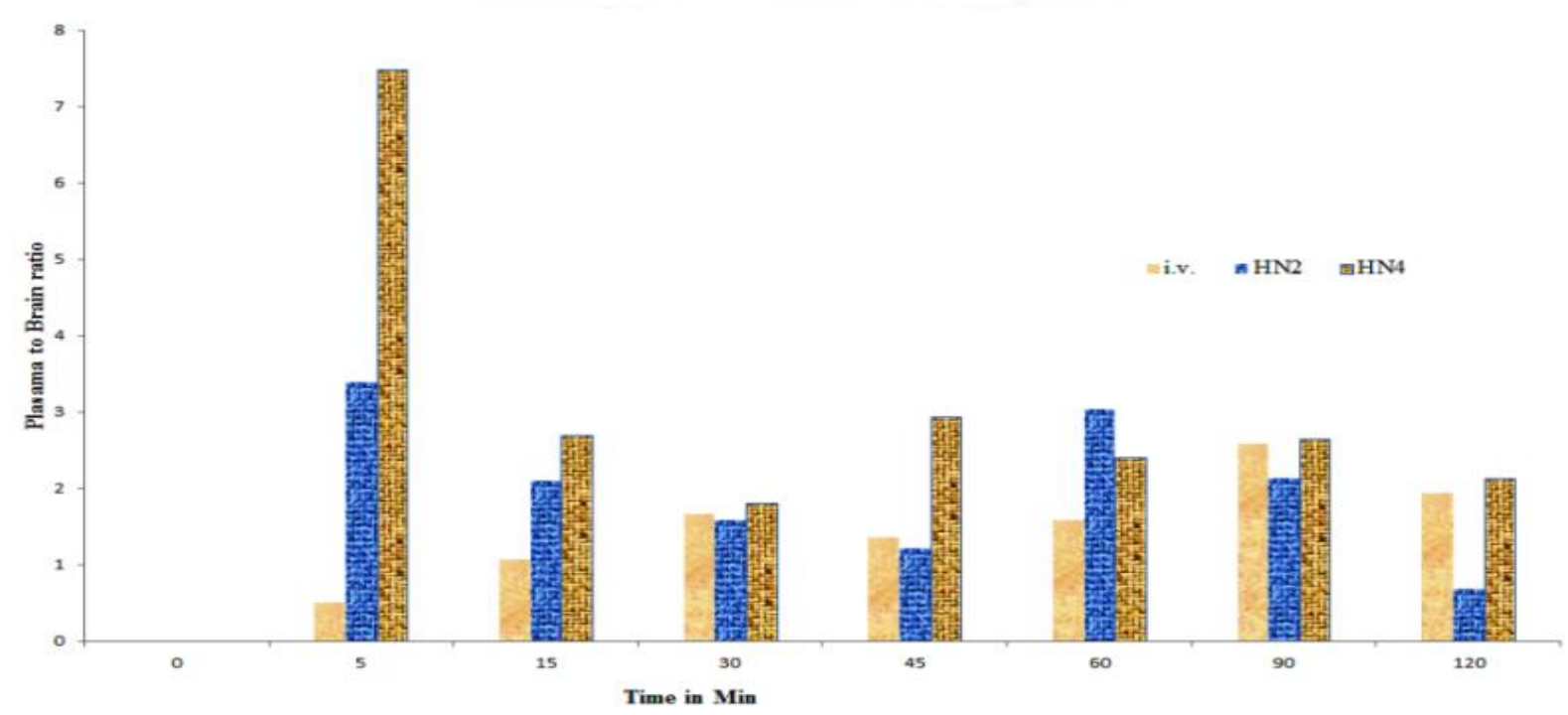

Figure 7: Showing a comparative Brain to Plasma Ratio of i.v. and intransal, HN2 and HN4 at different time point 


\section{CONCLUSION}

The study confirmed that there is direct nose to brain delivery of carbamazepine which can be advantageous for other CNS active drugs which shows side effects by oral or intravenous route. The results also show that the method of preparation was found suitable for making hybrid nanoparticles. The study also shows that Hybrid nanoparticles can be applied for acute conditions. It can be concluded that hybrid nanoparticles have a good targeting efficiency to the brain from nasal route.

Acknowledgement: The authors are thankful to department of Pharmaceutical Sciences Bhimtal campus and also thankful to IIT Roorki for providing TEM and zeta sizing facilities.

\section{REFERENCES}

1. Arya RKK, Juyal V, Kunwar N, Preparation of carbamazepine chitosan nanoparticles for improving nasal absorption, Journal of Drug Delivery and Therapeutics, 2015; 5(3):101-108. DOI: 10.22270/jddt.v5i3.1090

2. Serralheiro A, Gilberto A, Ana F, Amílcar F, Intranasal administration of carbamazepine to mice: a direct delivery pathway for brain targeting, Euro. J. of Pharma Sci, 2014, doi:dx. Doi.org/10. 1016/j.ejps. 2014. 04.019.

3. Gavini E, Hegge A B, Rassu G, Sanna V, Testa C, Pirisino G, Karlsen J, Giunchedi P Nasal administration of carbamazepine using chitosan microspheres: In-vitro/in-vivo studies, Int. J. Pharm, 2006; 307:9-15.

4. Barakat NS, Omar SA, Ahmed AAE, Carbamazepine uptake into rat brain following intra-olfactory transport, J. of Pharmacology, 2006; 58:63-72

5. Lee VH, Robinson JR, Controlled drug delivery fundamental and application $2^{\text {nd }}$ ed. revised and expanded. Dekker. New York; 2005. P. 29, 4.

6. $\mathrm{Ni} \mathrm{S}$, Nanoparticles carrying natural product for drug delivery, Journal of Drug Delivery and Therapeutics. 2017; 7(3):73-75 DOI: http://dx.doi.org/10.22270/jddt.v7i3.1425

7. Ravi MNV, Handbook of Particulate drug delivery, American Sci publishers, California, vol 2, 96-161.

8. Mygind N, Dahl R, Anatomy and physiology of the nasal cavities in health and disease, adv. drug del. Rev, 1998; 29:312.

9. Chein YW, Novel drug delivery systems, $2^{\text {nd }}$ ed. New York, Marcel Dekker Inc; 1993. P. 269-300.

10. Yildiz $\mathrm{O}$, Particulate carrier for nasal administration, handbook of particulate drug delivery, American scientific publisher, 2008. P. 143-161.

11. Sharma PK, Garg G, Salim M, Review on nasal drug delivery system with recent advancemnt, Int. J. Pharm. and Pharma. Sci., 2011; 3:6-11.
12. Nanosponges-a boon to the targeted drug delivery system, Yadav GV, Panchory HP, Journal of drug delivery and therapeutics, 2013; 3(4):151-155

13. Jadhav KR, Manoj N, Gambhire, Shaikh IM, Nasal drug delivery system-factors affecting and applications, Cur. Drug Therapy, 2007; 2:27-38.

14. Wong H, Rauth AM, Bendayan R, Wu XY, In-Vivo evaluation of a new polymer-lipid hybrid nanoparticles (Pln) formulation of doxorubicin in a murine solid tumor model. Eur. J. Pharma. Biopharma., 2007; 65:300-308.

15. Jian L, Li W, Shen YZ, Li YR, Wang YF, A novel polymer lipid nanoparticle for efficient nonviral gene delivery, Acta Pharm. Sinca, 2010; 31:509-514.

16. Wang J, Ballanco CO, Xu J, Yang W, Yu X, Preparation and characterization of vinculin-targeted polymer-lipid nanoparticle as intracellular delivery vehicle, Int. J. Nanomed., 2013; 3(8):39-46.

17. Sailor MJ, Park JH, Hybrid nanoparticles for detection and treatment of cancer, Adv. Mater., 2012; 24(28):3779-3802.

18. Hadinoto K, Sudaresan A, Cheow WS, Lipid-polymer hybrid nanoparticles as new generation therapeutic delivery platform: A review, Eur. J. of Pharma. Sci., 2013; 85(3):427443.

19. Gao J, Xia Y, Chen H, Yu Y, Song J, Li W, Qian W, Wang H Dai J, Guo Y, Polymer-lipid hybrid nanoparticles conjugate with anti-egf receptor antibody for target drug delivery to hepatocellular carcinoma, Nanomedicine 2014; 9(2):279-293

20. Pal SL, Jana U, Manna PK, Mohanta GP, Manavalan R, Nanoparticles: An overview of preparation and characterization, J. appl. Pharm. Sci. 2011; 1(6):228-234.

21. Vyas S P, Khar R K, Targeted And Controlled Drug Delivery: Novel Carrier System, Vallabh Prakashan, Delhi,2010 reprint

22. Lokhandwala H, Deshpande A, Deshpande S, Kinetic modeling and dissolution profiles comparison: an overview, Int. J. Pharm. Bio. Sci., 2013; 4(1):728 -73

23. Dash S, Murthy P N, Nath L, Chowdhury P, Kinetic modeling on drug release from controlled, Drug delivery systems, Acta Polo. Pharm. Drug Rese, 2010, 67(3)217-223.

24. Kapoor, Patel M, Vyas RB, Lad C, Lal B, Site specific drug delivery through nasal route using bioadhesive polymers,Journal of Drug Delivery and Therapeutics. 2015; $5(1): 1-9$

25. Bagger $\mathrm{M}$, Bechgaard $\mathrm{E}, \mathrm{A}$ microdialysis model to examine nasal drug delivery and olfactory absorption in rats using lidocaine hydrochloride as a model drug. Int. J. Pharm, 2004, 269, 311-322.

26. Tripathi D, Arya RKK, Pant M, Nanoparticles: A novel avenue in cancer, Int. J. Pharm. Sci. Rev. Res, 2016; 36(2):90-98. 